\title{
Impact of changes in physical activity on health-related quality of life among patients
} with COPD

\author{
C. Esteban*, J.M. Quintana\#, M. Aburto*, J. Moraza*, M. Egurrola*, \\ J. Pérez-Izquierdo*, S. Aizpiri*, U. Aguirre ${ }^{\#}$ and A. Capelastegui*
}

ABSTRACT: The aim of this study was to evaluate whether changes in regular physical activity (PA) affect health-related quality of life (HRQOL) among patients with chronic obstructive pulmonary disease (COPD).

611 patients (mean age $67.2 \pm 8.4$ yrs; forced expiratory volume in $1 \mathrm{~s} 49.7 \pm 14.6$ ) completed the St George's Respiratory Questionnaire (SGRQ), the Chronic Respiratory Questionnaire (CRQ) and the Medical Outcomes Short Form (SF-36) questionnaire. PA, defined as patients' selfreported regular walking times, was classified as low, moderate and high. After 5 yrs, 391 survivors completed these instruments again.

After adjustment for relevant confounders, patients who reported low PA at baseline and who increased their PA over the study period improved their SGRQ and CRQ scores by 15.9 and 8.7 points, respectively. Patients who moved from moderate to high PA improved their SGRQ scores by 18.4 and their CRQ scores by 14.8. Slightly smaller increases were observed for patients who maintained a high level of PA throughout the study period. Maintaining a low level of PA or decreasing PA over the study period was associated with a significant HRQoL decline.

Among COPD patients, a reduction in time spent engaging in PA or maintaining a low level may impair HRQoL, whereas an increase in PA can improve HRQoL parameters.

KEYWORDS: Chronic obstructive pulmonary disease, physical activity, quality of life

R egular physical activity (PA) has been shown to be beneficial in the general population for the primary and secondary prevention of cardiovascular disease, hypertension, type 2 diabetes mellitus, obesity, osteoporosis and some forms of cancer [1]. PA is associated with reduced mortality [2].These benefits apply to males and females, young and old [3]. Extreme levels of fitness are not required; engaging in moderate-intensity PA on most days of the week is generally sufficient [4]. Walking, an activity most people can perform, has been associated with nearly all of the benefits of regular PA [5].

Chronic obstructive pulmonary disease (COPD) is characterised by chronic airway obstruction that worsens over time. Although its primary impact is on the lungs, it also produces biochemical, structural and functional alterations throughout the body [6, 7]. These respiratory and systemic changes cause a progressive decline in health [8].

Quality of life is an important measure of health, particularly for older people and those suffering from a chronic disease. Thus, it is important to use health-related quality of life (HRQoL) tools that can evaluate the repercussions of a disease, such as COPD, on the patient. In patients with COPD, poor HRQoL has been associated with an increased likelihood of hospitalisation [9], hospital readmission [10] and mortality [11].

Data from cross-sectional studies show a direct association between HRQoL and PA in the general population, but the data are scarce and incomplete [12]. Even less information is available about this relationship among patients with COPD. As suggested in a population-based study, regular PA may counter the decline in HRQoL, in part, by reducing COPD-related hospital admissions and mortality and slowing the decline in forced expiratory volume in $1 \mathrm{~s}$ (FEV1) [13, 14].

We designed this study to determine the impact of changes in the level of PA, primarily low-intensity walking during leisure time, on HRQoL in a cohort of patients with COPD over a 5-yr period.
AFFILIATIONS

*Pneumology Dept, Hospital Galdakao-Usansolo, and \#Unidad de Investigación, CIBER Epidemiología y Salud Pública (CIBERESP), Hospital GaldakaoUsansolo, Galdakao, Spain.

\section{CORRESPONDENCE}

C. Esteban

Servicio de Neumología Hospital de Galdakao-Usansolo

Barrio Labeaga s/n. 48960 Galdakao

Bizkaia

Spain

E-mail: cristobal_esteban@yahoo.es

Received:

Feb 082009

Accepted after revision:

Dec 222009

First published online:

Jan 142010 


\section{METHODS}

\section{Subjects}

We recruited patients being treated for COPD at the outpatient clinics of Hospital Galdakao-Usansolo (Galdakao, Spain) between February 1998 and February 1999. Hospital GaldakaoUsansolo is a 400-bed teaching hospital in the Basque Country (northern Spain) that serves a population of 300,000 inhabitants. It belongs to the network of public hospitals of the Basque Health Care Service, which provides free unrestricted care to nearly $100 \%$ of the population. A respiratory rehabilitation programme was initiated in January 2008.

Consecutive patients were included in the study if they had been diagnosed with COPD for at least 6 months and had been receiving medical care at one of the hospital's outpatient facilities for at least 6 months. COPD had to be stable (no increase in respiratory symptoms or changes in treatment) for 6 weeks prior to enrolment. Other inclusion criteria were FEV1 $<80 \%$ of the predicted value, FEV1/forced vital capacity quotient $<70 \%$, and a negative bronchodilation test with an FEV1 change of $<200 \mathrm{~mL}$ and under $15 \%$ of the baseline value. The functional parameters used were those obtained following bronchodilation. Patients were not eligible for the study if they had been diagnosed with asthma, had extensive pulmonary tuberculosis or neoplastic processes, were suffering from psychiatric or neurological problems that might prevent effective collaboration, or had hearing or other problems that impeded accurate communication. Patients were also excluded if they had participated in a respiratory rehabilitation programme. Each patient was given detailed information about the study and provided verbal informed consent to take part. The study protocol was approved by the research committee of the Hospital Galdakao-Usansolo.

\section{Study protocol}

Patients who fulfilled the selection criteria were interviewed and underwent physical examination soon after recruitment. Spirometry was conducted following criteria from the Spanish Pneumology and Thoracic Surgery Society [15] with a MasterScope-PC spirometer (Erich Jaeger GmbH \& Co. KG, Wuerzburg, Germany). Theoretical values were those established by the European Community for Steel and Coal [16].

HRQoL was assessed at baseline using three instruments. The generic 36-item Medical Outcomes study Short Form (SF-36), including its physical component summary scale (PCSS) and mental component summary scale (MCSS) [17], and two diseasespecific questionnaires; the St. George's Respiratory Questionnaire (SGRQ) [18] and the Chronic Respiratory Questionnaire (CRQ) [19]. We used versions of all three instruments that had been validated in Spanish populations [20-22].

Normalised SF-36 values can be estimated to provide a reference value from the general population. To do this, each SF-36 component score is first standardised by using the mean and SD obtained from a Spanish population of males aged $>45 \mathrm{yrs}$ and then transformed to norm-based (mean \pm SD $50 \pm 10)$ scoring [23].

At the baseline personal interview, patients were asked about the types of PA they did. Special emphasis was placed on walking, with questions about time spent walking and distance covered, as described in previous studies [24]. The level of PA was defined as the time patients spent walking during their leisure time, and was classified into three categories: low (engaging in light PA such as walking for $<2 \mathrm{~h} \cdot$ week $^{-1}$ ); moderate (engaging in light PA such as walking for 2$4 \mathrm{~h} \cdot$ week $^{-1}$ ); and high (engaging in light PA such as walking for $>4 \mathrm{~h} \cdot$ week $^{-1}$ ), again as described in previous studies [13, 25-27]. Comorbidities were determined by reviewing patients' medical records for their clinical histories.

Patients were followed for up to 5 yrs. During this period, they continued to meet with their primary care physicians and respiratory specialists, and followed the recommendations and prescribed treatments. As is standard practice in our COPD clinics, all patients were urged to stop smoking, if applicable, and to engage in regular PA. Primary care physicians, respiratory specialists and other healthcare providers did not provide any interventions related to this study, and the research team did not intervene in patients' routine treatment or in the treatment of exacerbations.

5 yrs after enrolment the survivors were again interviewed, underwent spirometry and completed the three HRQoL questionnaires. As before, each patient was asked about the average time and distance they walked per day. Follow-up interviews were scheduled at outpatient clinics of our hospital during times when patients were clinically stable. Patients who developed a disease that could alter HRQoL or prevent them from completing the HRQoL questionnaires (e.g. cancer, Alzheimer's disease, etc.) and those who could not be interviewed personally, as well as those who were enrolled in a pulmonary rehabilitation programme during the followup period, were excluded from the study.

Vital status was determined by reviewing medical reports and examining the hospital database and public death registries. Deaths were considered to be confirmed if the record matched the subjects name, sex and date of birth. The cause of death in all cases was based on hospital reports and public death registries. For deaths that did not occurred in the hospital, the researchers telephoned relatives and the primary care physician. The research team analysed all data and established the causes of death.

\section{Statistical analysis}

Data are presented as mean \pm SD for continuous variables and frequencies and percentages for categorical variables.

For the comparison of survivors and nonsurvivors, we used the Chi-squared and Fisher's exact tests to evaluate associations among categorical variables and an unpaired t-test for continuous variables. Non-parametric tests were used when appropriate.

To evaluate possible correlations between the three levels of physical activity at baseline, the sociodemographic and clinical variables, and the SF-36, SGRQ and CRQ we used the ANOVA test with the Scheffee test for multiple comparisons and the non-parametric Kruskal-Wallis test. The same test was used to check for correlations among the changes in PA over the 5-yr study period. 
We also present the changes in PA over the study period, taking as a reference the normative data of the general population of the same age and sex.

We performed multivariate analysis by means of two generalised linear models. The dependent variables were the change in SGRQ scores over the study period and the change in CRQ scores, respectively. The main independent variable was the change in PA during the study (PA categories at 5 yrs versus PA categories at basal time) adjusted by age, FEV1, previous hospital admissions, level of dyspnoea, comorbidities and mental health status (measured by the SF-36 MCSS) at baseline, and by baseline HRQoL as measured by SGRQ or CRQ scores, respectively, in each corresponding model.

A p-value $<0.05$ for effects were considered significant, unless otherwise stated. All statistical analyses were performed using SAS for Windows statistical software (version 8.2; SAS Institute Inc., Carey, NC, USA).

\section{RESULTS}

A total of 611 patients fulfilled the study criteria and completed the baseline questionnaires. After 5 years of follow-up, $445(72.8 \%)$ patients were still alive. The causes of death included exacerbations of COPD $(n=81)$, other respiratory causes $(n=16)$, cardiovascular or cerebrovascular disease $(n=40)$ and other causes $(n=26)$, mainly various neoplasms. No clear cause of death was recorded for three patients (sudden death). The baseline characteristics of study participants who survivors and those who died are shown in table 1.

Among the 445 survivors, 54 patients were not included in the final analysis due to active neoplasms $(n=25)$ or they had moved out of the area $(n=19)$, had developed psychiatric disorders or Alzheimer's disease $(n=7)$, had become blind $(n=1)$ and could not be located $(n=2)$. The characteristics of these 54 patients were not different from the 391 survivors in terms of level of PA or FEV1 \% pred, dyspnoea, smoking habits, comorbidities or HRQoL.

\section{TABLE 1 Baseline characteristics of study participants by survival status over a 5-yr follow-up period}

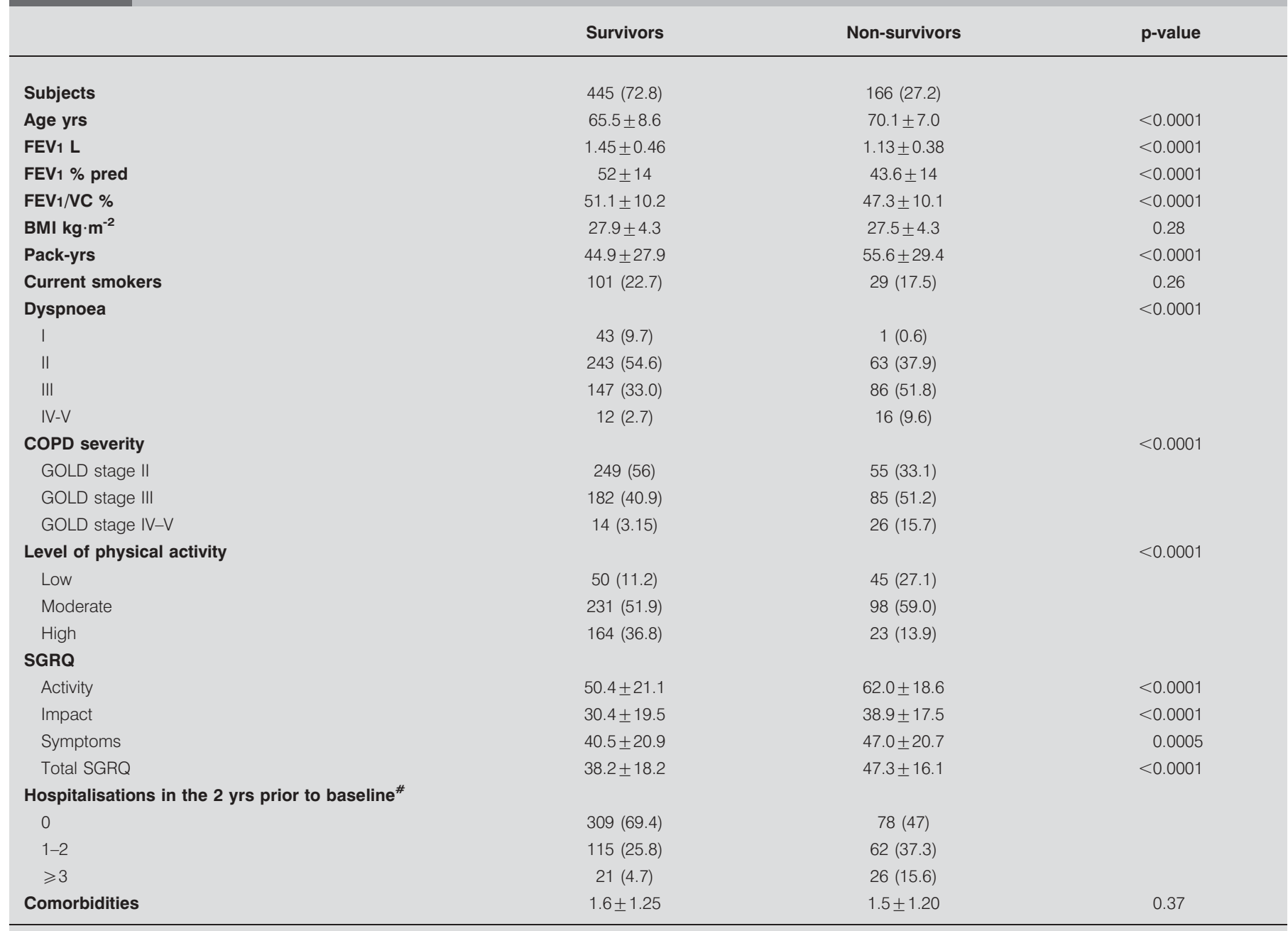

Data are presented as $n(\%)$ or mean \pm SD, unless otherwise stated. FEV1: forced expiratory volume in $1 \mathrm{~s}$; \% pred: \% predicted; VC: vital capacity; BMI: body mass index; COPD: chronic obstructive pulmonary disease; GOLD: Global Initiative for Chronic Obstructive Lung Disease; SGRQ: Saint George's Respiratory Questionnaire. ${ }^{\#}: \mathrm{p}<0.0001$. 
At baseline, patients who reported lower levels of PA had more dyspnoea, were more likely to have been admitted to the hospital for an exacerbation of COPD in the 2 yrs prior to enrolment, had more comorbidities and had lower FEV1 than those with moderate or high levels of PA. There were no significant differences in the other variables between the three PA groups (table 2).

A number of differences in HRQoL were observed across PA categories. At baseline there were statistically significant differences in SGRQ scores across the three PA categories in most areas. Differences in CRQ scores were mainly observed between the low PA and high PA groups. With regard to SF-36 scores, differences were generally observed between the low PA category and the two higher categories (table 3). Statistically significant differences between the three groups were observed in the area of physical functioning.

After 5 yrs of follow-up, 254 patients reported the same level of PA as that reported at baseline, 75 reported less PA and 62 reported increased PA. The total SGRQ for the cohort was $38.7 \pm 20.8$ and the total CRQ was $100.7 \pm 27.4$. The distribution of the changes in PA is reflected in table 4. In general, maintaining a low level of PA or decreasing PA over the study period was associated with a statistically significant decline in HRQoL, as measured by the SGRQ and CRQ. Maintaining a high level of PA or increasing PA was associated with an improvement in HRQoL, with greater increases in SGRQ than CRQ scores. In the SF-36 PCSS, a decline was observed across all categories of PA, with the largest changes observed among patients who reported a lower PA level after 5 yrs of follow-up. In the MCSS, there was a decline in HRQoL among patients with low PA at baseline and after 5 yrs of follow-up, as well as in those who reported a moderate level of PA at baseline and a low level after 5 yrs (table 4).

Changes in PA over the study period, taking the normative data of the general population of the same age and sex as a reference, showed that those who improved their PA over the course of the 5-yr follow-up period preserved their SF-36 PCSS values and slightly improved their MCSS. However, in general, they did not reach the levels observed for the general population (fig. 1).

\section{TABLE 2 \\ Baseline characteristics of the 391 survivors who completed the three health-related quality of life instruments after 5 yrs} of follow-up

\begin{tabular}{|c|c|c|c|c|}
\hline & \multicolumn{3}{|c|}{ Level of physical activity } & \multirow[t]{2}{*}{ p-value } \\
\hline & Low $^{\#}$ & Moderate & $\mathrm{High}^{+}$ & \\
\hline Subjects $\mathbf{n}$ & 41 & 207 & 143 & \\
\hline Age yrs & $65.8 \pm 8.6^{+}$ & $67.3 \pm 7.1^{+}$ & $61.9 \pm 9.7^{\# \cdot}$ & $<0.0001$ \\
\hline $\mathrm{FEV}_{1} \mathrm{~L}$ & $1.36 \pm 0.50$ & $1.43 \pm 0.44^{+}$ & $1.56 \pm 0.48^{\bullet}$ & 0.01 \\
\hline FEV $_{1} \%$ pred & $51.4 \pm 16.3^{\bullet++}$ & $51.9 \pm 13.6^{\#,+}$ & $53.3 \pm 13.7^{\# \cdot \bullet}$ & $<0.0001$ \\
\hline BMI & $28.2 \pm 6.7$ & $27.9 \pm 3.9$ & $28.0 \pm 4.1$ & 0.94 \\
\hline Pack-yr smoking habit & $46.4 \pm 28.2$ & $43.7 \pm 30.2$ & $44.0 \pm 25.1$ & 0.85 \\
\hline Comorbidities & $2.2 \pm 1.4^{\bullet++}$ & $1.7 \pm 1.2^{\#}$ & $1.5 \pm 1.2^{\#}$ & 0.003 \\
\hline Dyspnoea & & & & $<0.0001$ \\
\hline 1 & 0 & $7(3.4)$ & $32(22.4)$ & \\
\hline$\|$ & $11(26.8)$ & $117(56.5)$ & $85(59.4)$ & \\
\hline III & $26(63.4)$ & $80(38.6)$ & $25(17.5)$ & \\
\hline IV-V & $4(9.8)$ & $3(1.5)$ & $1(0.7)$ & \\
\hline GOLD stage & & & & 0.90 \\
\hline I & $21(51.2)$ & $117(56.5)$ & $85(59.4)$ & \\
\hline$\|$ & $19(46.3)$ & $84(40.6)$ & $54(37.8)$ & \\
\hline III-IV & $1(2.4)$ & $6(2.9)$ & $4(2.8)$ & \\
\hline Smoking status & & & & 0.30 \\
\hline Current smoker & $7(17.7)$ & $39(18.8)$ & $39(27.3)$ & \\
\hline Ex-smoker & $31(75.6)$ & $158(76.3)$ & $99(69.2)$ & \\
\hline Never-smoker & $3(7.3)$ & $10(4.8)$ & $5(3.5)$ & \\
\hline $\begin{array}{l}\text { Hospitalisations for COPD exacerbation } \\
\text { in the } 2 \text { yrs prior to enrolment }\end{array}$ & & & & 0.001 \\
\hline 0 & $22(53.6)$ & $136(65.7)$ & 117 (81.8) & \\
\hline 1 & $11(26.8)$ & 46 (22.2) & 19 (13.3) & \\
\hline$\geqslant 2$ & $8(19.5)$ & $25(12.1)$ & $7(4.9)$ & \\
\hline
\end{tabular}

Data are presented as mean \pm SD or $n$ (\%), unless otherwise stated. FEV1: forced expiratory volume in 1 s; \% pred: \% predicted; BMI: body mass index; GOLD: Global Initiative for Chronic Obstructive Lung Disease; COPD: chronic obstructive pulmonary disease. ${ }^{*}$ : Iow physical activity; ${ }^{\bullet}$ : moderate physical activity; ${ }^{+}$: high physical activity. ${ }^{*}$, and ${ }^{+}$indicate differences among the three physical activity categories using Scheffé test for multiple comparisons for continuous variables at $\mathrm{p}<0.05$. Chisquared tests were used for the comparison of proportions among the three physical activity categories. 


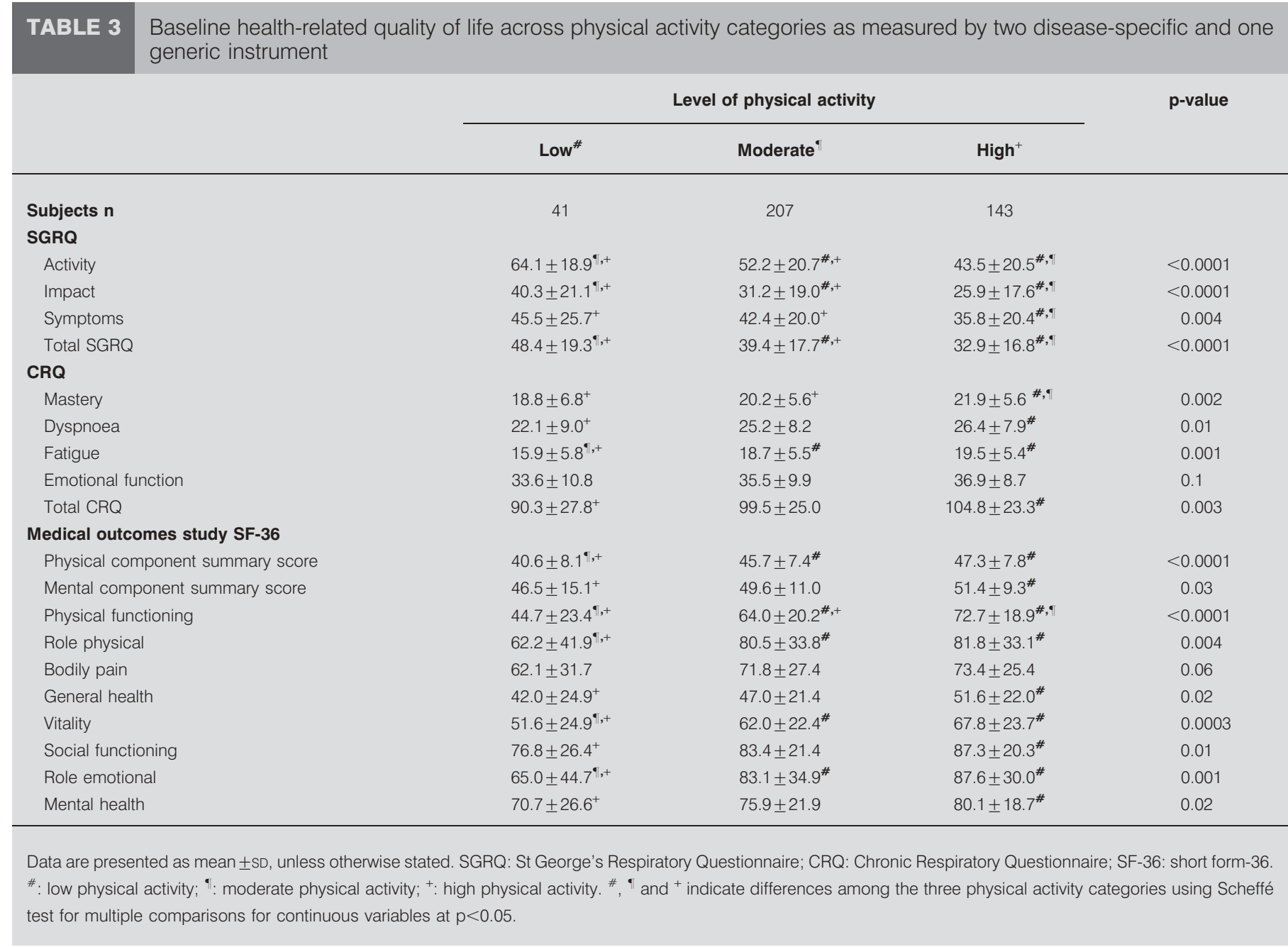

Multivariate analysis of two different models used the total SGRQ score (model 1) and the total CRQ score (model 2) as separate dependent variables, and changes in the reported level of PA over the 5-yr study period as the main independent variable (table 5). Changes in the self-reported level of PA over the course of the study were independently associated with changes in HRQoL in the two models. Using the patients who reported high levels of PA at baseline and after 5 yrs (high/ high) as a reference group, patients who reported low levels of PA at baseline and after 5 yrs (low/low) experienced a decline in HRQoL (an increase of 16.9 units in the total SGRQ score). A similar decline was observed among patients who reported a decline in PA after 5 yrs of follow-up (moderate/low and high/moderate-low). When patients in the lowest category of baseline and 5-yr PA (low/low) were used as a reference group, improvements in HRQoL (as measured by decreases in SGRQ scores) were observed among patients who increased their PA from baseline or who maintained moderate or high levels of PA (table 5). Patients who changed their PA level from low to moderate/high had almost 16 less points in the SGRQ than those who maintained their PA as low at 5 yrs. Maintaining the PA level as moderate for 5 yrs improved the SGRQ score in 10 units; changing from moderate to high or maintaining at high level improved the total domain of the
SGRQ in $>16$ units in comparison to patients who stayed at low PA level during the study. The magnitude of the improvement in HRQoL was similar regardless of the baseline level of PA. Other variables that were evaluated, such as body mass index and pack-years of smoking, did not influence the change in HRQoL. While the mean number of comorbidities increased from 1.7 at baseline to 1.9 after 5 yrs of follow-up $(p<0.01)$, this did not have a significant impact in the multivariate analysis.

\section{DISCUSSION}

Among patients with COPD who were not participating in a structured respiratory rehabilitation programme, changes in the level of leisure-time PA (as measured by the average time spent walking each day) was strongly associated with HRQoL. Patients who engaged in low levels of PA over the 5-yr study period and those whose level of PA decreased experienced significant declines in HRQoL. In contrast, HRQoL improved in patients who maintained moderate or high levels of PA and those who increased their PA. We focused on a specific PA, walking, since it is a very common leisure-time activity that can easily be performed by older individuals and those with medical conditions, such as COPD. 


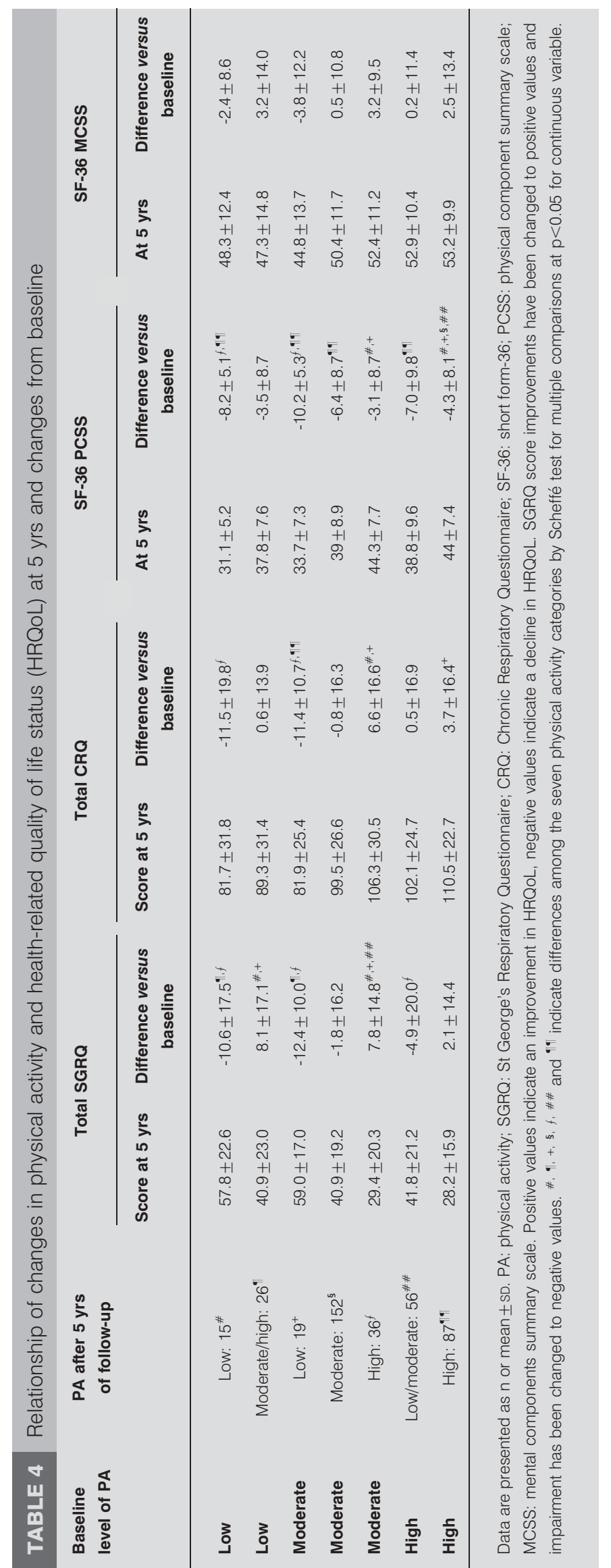

In our univariate analysis, patients who engaged in low levels of PA had more dyspnoea, more comorbidities, more hospital admissions in the previous $2 \mathrm{yrs}$, lower FEV1 and poorer HRQoL than those with high PA. This corresponds to the results of a cross-sectional study that established comorbidities such as diabetes, HRQoL and home oxygen therapy as determinants of low PA [28]. It also corresponds with another study in which patients who had been hospitalised during the previous year for an exacerbation of COPD reported lower levels of PA than those who had not been hospitalised for a COPD exacerbation [29].

In a population-based cohort study, GARCIA-AYMERICH et al. [13] reported that patients with even relatively low levels of PA (walking or pedalling for $2 \mathrm{~h}$ per week) had a $28 \%$ lower risk of hospitalisation due to COPD than those with little or no PA. All-cause mortality and respiratory mortality were also lower. The investigators did not, however, observe a dose-related response between the level of PA and either hospitalisations or respiratory mortality.

Although preventing respiratory-related mortality is of utmost importance, improving quality of life should not be overlooked. In our study, patients with COPD who maintained low levels of PA or who decreased their PA over the study period experienced declines in HRQoL, whereas those who increased their PA or maintained moderate or high levels of PA demonstrated improvements in HRQoL in the specific HRQoL questionnaires and these changes were both statistically and clinically significant (increase of $>4$ units in the SGRQ).

To the best of our knowledge, this is the first study to evaluate the impact of changes in leisure-time PA on HRQoL among patients with COPD who are not taking part in a respiratory rehabilitation programme. Respiratory rehabilitation programmes have proved beneficial in improving health and HRQoL among patients with COPD [30]. However, these programmes offer multiple features in addition to physical training that probably contribute to the benefits. To date, it has not been established which aspects of respiratory rehabilitation programmes have the greatest impact on health, mortality and HRQoL.

It is probable that PA improves HRQoL among patients with COPD for the same multifactorial reasons that it benefits the general population. PA improves cardiac function and increases maximum oxygen uptake. It has positive effects on blood pressure, lipid profiles and clotting mechanisms. It is also associated with healthier lifestyles. Among patients with COPD, regular PA improves peripheral muscle function [31]. It also reduces hospital admissions [13], which have been shown to impair HRQoL [32].

Among patients with COPD, HRQoL appears to evolve differently when evaluated with a generic questionnaire (SF36) than when it is evaluated using a disease-specific questionnaire. At the beginning of our study, patients reporting low levels of PA had the lowest HRQoL, as measured by PCSS scores. Other investigators have observed similar associations among patients with COPD [28]. It has also been observed in healthy adults aged 60-89 yrs [33], where individuals who engaged in PA of moderate intensity for $>1 \mathrm{~h}$ per week had better HRQoL in five areas of the SF-36, including the four physical areas. In our study, the evolution of PCSS scores over 

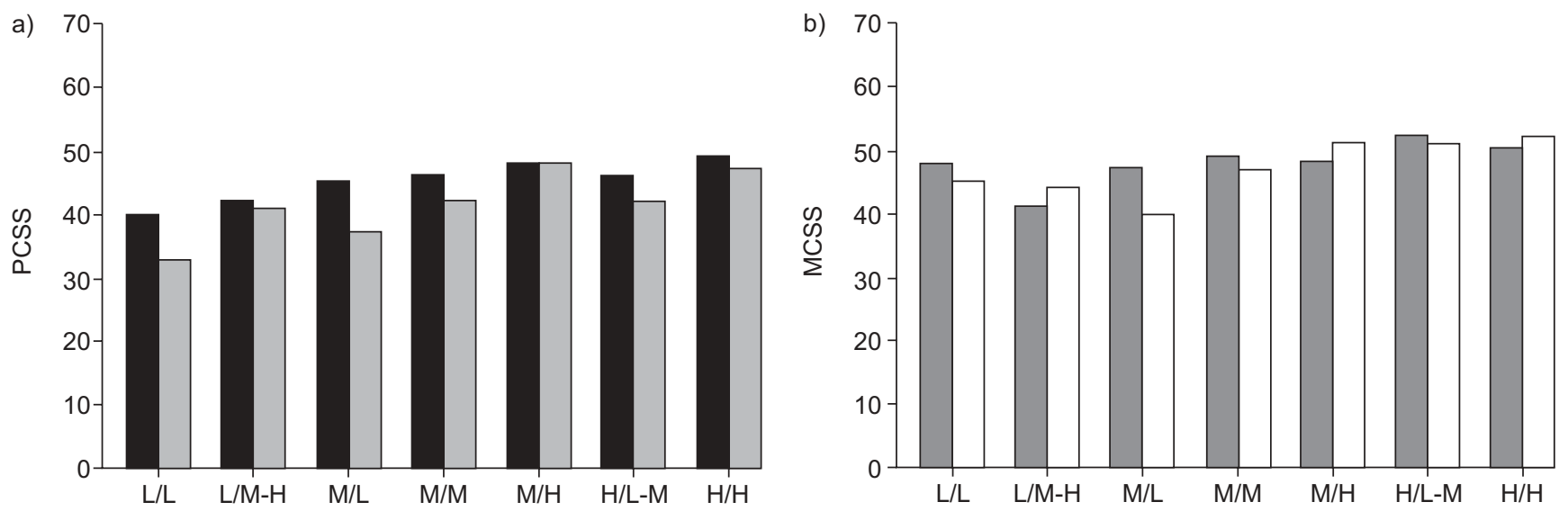

FIGURE 1. a) Evolution of short form (SF)-36 physical component summary scale (PCSS) in relation to changes in physical activity. b) Evolution of short form (SF)-36 mental component summary scale (MCSS) in relation to changes in physical activity. L/L: from low to low physical activity; L/M-H: from low to moderate or high physical activity; M/L: from moderate to low physical activity; M/M: from moderate to moderate physical activity; M/H: from moderate to high physical activity; H/L-M: from high to low

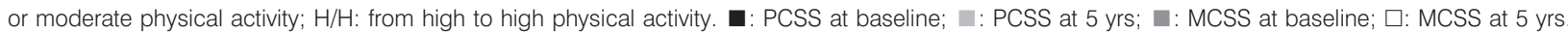

5 yrs of follow-up showed declines in HRQoL at all levels of PA compared to both baseline and the general population. However, the decline in HRQoL was greater for patients who maintained low levels of PA or who decreased the amount of time engaged in PA.

Among patients with COPD, we are unaware of any study examining the impact of changes in PA on HRQoL measured with a generic questionnaire. In a study conducted among 3,891 healthy individuals followed for 3 yrs, higher PA during leisure time was associated with an improvement in HRQoL, especially in the mental components of SF-36, although the strength of the association was limited and the magnitude of the change would probably have no clinical significance [34]. In our study, patients with higher levels of PA had MCSS scores similar to those in the general population. Among patients with low and moderate PA who decreased the amount time engaged in PA over the 5-yr study period, MCSS scores declined from baseline, with an even more striking difference compared with the general population. In our study, the loss of HRQoL found in the PCSS probably reflects the natural decline in HRQoL with the passing of time. This tends to be especially pronounced among populations with a chronic disease, such as COPD, which has substantial respiratory and systemic consequences. This corresponds to the overall trend toward declining HRQoL over time as measured with a generic instrument, such as the

TABLE 5 Multivariate analysis of physical activity (PA) changes and health-related quality of life (HRQLL) changes from baseline to 5-yr follow-up adjusted by relevant variables

Level of PA baseline/5 yrs

\begin{tabular}{l} 
\\
\hline Low/low \\
Low/moderate-high \\
Moderate/low \\
Moderate/moderate \\
Moderate/high \\
High/moderate-low \\
High/high
\end{tabular}

SGRQ: St George's Respiratory Questionnaire; CRQ: Chronic Respiratory Questionnaire. Positive values indicate an improvement in HRQoL, negative values indicate a decline in HRQoL. SGRQ score improvements have been changed to positive values and impairment has been changed to negative values. Multivariate models where the dependent variables were the change in the total SGRQ score (model 1) and the total CRQ score (model 2) from baseline to 5 yrs. The main independent variable in model 1 was the change in PA over the 5 -yr period, adjusted by age, dyspnoea, forced expiratory volume in $1 \mathrm{~s}$ (\% pred), hospital admissions for chronic obstructive pulmonary disease (COPD) exacerbations in the 2 yrs prior to enrolment, comorbidities and mental health status (measured by the short form (SF)-36 Mental Component Summary Scale (MCSS1)) at baseline, as well as by baseline HRQoL measured by the total SGRQ score. The main independent variable in model 2 was the change in PA over the 5-yr study period adjusted by hospital admissions for COPD exacerbations in the 2 yrs prior to enrolment and baseline mental health status (measured by the SF36 MCSS1), as well as by the baseline HRQoL levels at the beginning of the study measured by the total CRQ score. The reference group for comparison in both models were those who had a low level of PA at the beginning and also at 5 yrs of follow-up. 
SF-36. Such changes are more pronounced in older age groups and in the physical domains [35].

The strengths of our study include the large size of the cohort (611 patients), the fact that patients were recruited from general outpatient clinics, the long follow-up period (5 yrs) and the use of three different questionnaires; two of which are designed specifically for patients with COPD. Another strength of our study is that the patients did not receive any special studyrelated treatments or interventions, such as a rehabilitation programme during the follow-up period. Instead, they continued to follow the controls and treatments prescribed by their healthcare providers, none of whom received any direction regarding treatment from the study authors. In addition to comparing levels of PA with HRQoL, we tracked changes in HRQoL over time and compared these to changes in a general population. We conducted statistical adjustments in an effort to prevent confounding factors from biasing the results.

Limitations of the study must also be noted. Since confounding and reverse causation are possible in this study, no causality or directionality of the findings can be inferred. We used a questionnaire to establish the level of PA rather than a direct measure, such as an accelerometer or pedometer. At the time this study was performed, the use of more objective measures of assessment of PA, such as triaxial accelerometers was not common; however, they are becoming the new standard for PA measurements in studies with larger samples [36]. Valid, questionnaires that measure PA may be useful as a group estimate, but their lack of accuracy and large individual variability indicate that relying on them on an individual basis is not recommended [37]. Self-reports by study subjects may overestimate or underestimate PA. However, the scale we used, apart from having been previously validated and used in other studies of patients with COPD [13, 25], established differences between the three levels of PA in the physical functioning domain of the SF-36, which would support the discriminatory capacity of this instrument.

We also focused on walking as a measure of PA, and did not include other activities such as time spent doing domestic chores. While it is possible that other types of PA may influence HRQoL, in our environment walking is the most common form of leisure-time PA. Another limitation of our study is the small sample size in some of the categories of PA change during the follow-up period. Although we were able to detect statistical and clinically significant changes in some of these categories, due to the small sample size those results should be interpreted cautiously. As expected, patients who died during the follow-up period had poorer PA and HRQoL at baseline than those who survived. This biases the results which are based on survival, since we were not able to examine the changes in PA and HRQoL among those who died. Some non-studied factors may also play a role in the interaction between PA and quality of life in COPD.

The patient population was almost entirely comprised of males (97\%). Similar sex distributions have been observed in other studies performed in Spain [38]. However, this sex distribution could be different as has been shown in a recent epidemiological study [39]. Generalising our results to the female population would not be appropriate.
In conclusion, we found that patients with COPD who maintained a low level of PA or who engaged in less PA over time were more likely to experience a significant impairment in HRQoL. Patients who increased their levels of PA or who maintained them at a higher level, in a low intensity activity such as walking for $\geqslant 2 \mathrm{~h}$ per week, improved or maintained their HRQoL levels, regardless of the baseline level of PA. This suggests that patients with COPD could significantly improve their HRQoL with only a minor increment in their leisure-time PA and without any special supervision. In addition to including patients with COPD in respiratory rehabilitation programmes, interventions that increase PA, such as daily walking, could yield important improvements in HRQoL among patients with COPD. Future studies should incorporate more objective measures of PA, such as data from multiaxial accelerometers and evaluation of exercise capacity, include larger sample sizes of different PA categories and provide longer follow-up.

\section{SUPPORT STATEMENT}

This study was partially supported by the Fondo de Investigación Sanitaria (Madrid, Spain) (grant no. 97/0326) and the Research Committee Hospital Galdakao-Usansolo (Galdakao, Spain).

\section{STATEMENT OF INTEREST}

None declared.

\section{REFERENCES}

1 Kesaniemi YK, Danforth E Jr, Jensen MD, et al. Dose-response issues concerning physical activity and health: an evidence-based symposium. Med Sci Sports Exerc 2001; 33: Suppl. 6, S351-S358.

2 Blair SN, Kohl HW, Paffenbarger RS, et al. Physical fitness and all cause mortality. A prospective study of healthy men and women. JAMA 1989; 273: 1093-1098.

3 Siscovick DS, Fried L, Mittelmark M, et al. Exercise intensity and subclinical cardiovascular disease in the elderly. The Cardiovascular Health Study. Am J Epidemiol 1997; 145: 977-986.

4 Haskell WL, Lee IM, Pate RR, et al. Physical activity and public health: updated recommendation for adults from the American College of Sports Medicine and the American Heart Association. Med Sci Sports Exerc 2007; 39: 1423-1434.

5 Manson JE, Greenland P, LaCroix AZ, et al. Walking compared with vigorous exercise for the prevention of cardiovascular events in women. N Engl J Med 2002; 347: 716-725.

6 Celli BR, MacNee W, Agusti A, et al. Standards for the diagnosis and treatment of patients with COPD: a summary of the ATS/ERS position paper. Eur Respir J 2004; 23: 932-946.

7 Gross NJ. Extrapulmonary effects of COPD. Curr Opin Pulm Med 2001; 7: 84-92.

8 Ferrer M, Alonso J, Morera J, et al. Chronic obstructive pulmonary disease stage and health related quality of life. Ann Intern Med 1997; 127: 1072-1079.

9 Fan SF, Curtis JR, Tu SP, et al. Using quality of life to predict hospitalizations and mortality in patients with chronic obstructive pulmonary disease. Chest 2002; 122: 429-436.

10 Osman LM, Godden DJ, Friend JAR, et al. Quality of life readmission in patients with chronic obstructive pulmonary disease. Thorax 1997; 52: 67-71.

11 Domingo-Salvany A, Lamarca R, Ferrer M, et al. Health related quality of life and mortality in male patients with chronic obstructive pulmonary disease. Am J Respir Crit Care Med 2002; 166: 680-685. 
12 Bize R, Johnson JA, Plotnikoff RC. Physical activity level and health related quality of life in the general adult population: a systematic review. Prev Med 2007; 45: 401-415.

13 Garcia-Aymerich J, Lange $\mathrm{P}$, Benet $\mathrm{M}$, et al. Regular physical activity reduces hospital admission and mortality in chronic obstructive pulmonary disease: a population based cohort study. Thorax 2006; 61: 772-778.

14 Garcia-Aymerich J, Lange P, Benet M, et al. Regular physical activity modifies smoking-related lung function decline and reduces risk of chronic obstructive pulmonary disease: a population-based cohort study. Am J Respir Crit Care Med 2007; 175: 458-463.

15 Sanchis J. [Normativa para la espirometría forzada: Grupo de trabajo de la SEPAR para la práctica de la espirometría clínica.] Arch Bronconeumol 1989; 25: 132-142.

16 Quanjer PH, Tammeling GJ, Cotes JE, et al. Lung volumes and forced ventilatory flows. Report working party standardization of lung function test. European Community for Steel and Coal. Official statement of the European Respiratory Society. Eur Respir J 1993; 6: Suppl. 16, s5-s40.

17 McHorney CA, Ware JE, Jr, Raczek AE. The MOS 36-Item ShortForm Health Survey (SF-36): II. Psychometric and clinical tests of validity in measuring physical and mental health constructs. Med Care 1993; 31: 247-263.

18 Jones PW, Quirk FH, Baveystock CM. The St George's Respiratory Questionnaire. Respir Med 1991; 85: Suppl. B, 25-31.

19 Guyatt GH, Berman LB, Townsend M, et al. A measure of quality of life for clinical trials in chronic lung disease. Thorax 1987; 42: 773-778.

20 Alonso J, Prieto L, Ferrer M, et al. Testing the measurement properties of the Spanish version of the SF-36 health survey among male patients with chronic obstructive pulmonary disease. J Clin Epidemiol 1998; 51: 1087-1094.

21 Ferrer M, Alonso J, Prieto L, et al. Validity and reliability of the St George's Respiratory Questionnaire after adaptation to a different language and culture: the Spanish example. Eur Respir J 1996; 9: 1160-1166.

22 Güell R, Casan P, Sangenís M, et al. Quality of life in patient with chronic respiratory disease: the Spanish version of the chronic respiratory questionnaire (CRQ). Eur Respir J 1998; 11: 55-60.

23 Alonso J, Regidor E, Barrio G, et al. [Population reference values of the Spanish version of the Health Questionnaire SF-36.] Med Clin (Barc) 1998; 111: 410-416.

24 Esteban C, Quintana JM, Aburto M, et al. A simple score for assessing stable chronic obstructive pulmonary disease. QJM 2006; 99: 751-759.
25 Schnohr P, Scharling H, Jensen JS. Changes in leisure time physical activity and risk of death: an observational study of 7000 men and women. Am J Epidemiol 2003; 158: 639-644.

26 Saltin B, Grimby G. Physiological analysis of middle-aged and old former athletes: comparison with still active athletes of the same ages. Circulation 1968; 38: 1104-1115.

27 Saltin B. Physiological effects of physical conditioning. In: Hansen AT, Schnohr P, Rose G, eds. Ischaemic Heart Disease: the Strategy of Postponement. Chicago, Year Book Medical Publishers, 1977; pp. 104-115.

28 Garcia-Aymerich J, Félez MA, Escarrabil J, et al. Physical activity and its determinants in severe chronic obstructive pulmonary disease. Med Sci Sport Exerc 2004; 36: 1667-1673.

29 Pitta F, Troosters T, Probst VS, et al. Physical activity and hospitalization for exacerbation of COPD. Chest 2006; 129: 536-544.

30 Griffiths TL, Burr ML, Campbell IA, et al. Results at 1 year of outpatient multidisciplinary pulmonary rehabilitation: a randomised controlled trial. Lancet 2000; 355: 362-368.

31 Casaburi R. Skeletal muscle dysfunction in chronic obstructive pulmonary disease (review). Med Sci Sports Exerc 2001; 33: Suppl. 7, S662-S670.

32 Miravitlles M, Ferrer M, Pont A, et al. Effect of exacerbations on quality of life in patients with chronic obstructive pulmonary disease: a 2 year follow up study. Thorax 2004; 59: 387-395.

33 Acree LS, Longfors J, Fjeldstad AS, et al. Physical activity is related to quality of life in older adults. Health Qual Life Outcomes 2006; 4: 37.

34 Tessier S, Vuillemin A, Bertrais S, et al. Association between leisure-time physical activity and health-related quality of life changes over time. Prev Med 2007; 44: 202-208.

35 Hopman WM, Berger C, Joseph L, et al. The natural progression of health-related quality of life: results of a five-year prospective study of SF-36 scores in a normative population. Qual Life Res 2006; 15: 527-536.

36 Hagströmer M, Oja P, Sjöström M. Physical activity and inactivity in an adult population assessed by accelerometry. Med Sci Sports Exerc 2007; 39: 1502-1508.

37 Pitta F, Troosters T, Probst VS, et al. Quantifying physical activity in daily life with questionnaires and motion sensors in COPD. Eur Respir J 2006; 27: 1040-1055.

38 Soler-Cataluña JJ, Martinez-Garcia MA, Román Sánchez P, et al. Severe acute exacerbations and mortality in patients with chronic obstructive pulmonary disease. Thorax 2005; 60: 925-931.

39 Miravitlles M, Soriano JB, García-Río F, et al. Prevalence of COPD in Spain: impact of undiagnosed COPD on quality of life and daily life activities. Thorax 2009; 64: 863-868. 